\title{
Scaling behavior of the crossover to short-stack regimes of Josephson vortex lattices in $\mathrm{Bi}_{2} \mathrm{Sr}_{2} \mathrm{CaCu}_{2} \mathrm{O}_{8+\delta}$ stacks
}

\author{
I. Kakeya, $,{ }^{1,2}, *$ Y. Kubo, ${ }^{2}$ M. Kohri, ${ }^{2}$ M. Iwase, ${ }^{2}$ T. Yamamoto, ${ }^{2}$ and K. Kadowaki ${ }^{2}$ \\ ${ }^{1}$ Department of Electronic Science and Engineering, Kyoto University, Nishikyo, Kyoto 615-8510, Japan \\ ${ }^{2}$ Institute of Materials Science, University of Tsukuba, Tsukuba, Ibaraki 305-8573, Japan \\ (Received 21 November 2008; revised manuscript received 30 April 2009; published 8 June 2009)
}

\begin{abstract}
We report the systematic investigations of the oscillation of the Josephson vortex (JV) flow resistance in $\mathrm{Bi}_{2} \mathrm{Sr}_{2} \mathrm{CaCu}_{2} \mathrm{O}_{8+\delta}$ microfabricated junctions with various geometries and superconducting anisotropy parameters. As the applied magnetic field parallel to the $a b$ plane is increased, oscillation with a period corresponding to a half flux quantum par atomic Josephson junction changes to oscillation with a doubled period. This crossover is scaled by both the junction length and the anisotropy parameter indicating that the bulk inductive coupling that favors the triangular JV lattice is replaced with the surface deformation energy as the dominant interaction for a JV lattice. These results suggest that the in-phase square JV lattice is pronounced at a higher magnetic field in a smaller and more anisotropic sample.
\end{abstract}

DOI: 10.1103/PhysRevB.79.212503

PACS number(s): 74.50.+r, 74.25.Qt, 74.81.Fa, 74.72.Hs

Highly anisotropic high- $T_{c}$ superconductors (HTSC) such as $\mathrm{Bi}_{2} \mathrm{Sr}_{2} \mathrm{CaCu}_{2} \mathrm{O}_{8+\delta}(\mathrm{Bi} 2212)$ are well described as stacks of weakly coupled Josephson junctions (JJ's) that consist of $\mathrm{CuO}_{2}$ superconducting and charge reservoir block layers, ${ }^{1,2}$ referred to as intrinsic Josephson junctions (IJJ's). Not only the inductive coupling ${ }^{3}$ but also the capacitive coupling ${ }^{4}$ between the stacked Josephson junctions are responsible for such peculiar properties of IJJ's as multiple branches in the current-voltage $(I V)$ characteristics and the longitudinal Josephson plasma mode. ${ }^{5}$ Rich collective excitation phenomena attributed to these couplings among identical stacked junctions ${ }^{6}$ can be adopted for investigating physics of HTSC and also promise us potentials for future device applications.

In the magnetic field parallel to the $a b$ plane, where Josephson vortices (JV's) quantized as a unit of $\phi_{0}=h / 2 e$ are induced, various static and dynamic arrangements of JV's are expected. ${ }^{7,8}$ The variety of phenomena is given by the couplings between the adjacent JJ's, which make IJJ's a sharp contrast with artificially stacked JJ's. The other characteristic features of the JV system in parallel magnetic fields have been found in the $I V$ characteristics, ${ }^{9,10}$ the Josephson plasma resonance, ${ }^{11}$ etc.

Recently, Ooi et al. ${ }^{12}$ found a periodic oscillation of the JV flow resistance with a period of $\phi_{0} / 2$ in Bi2212 IJJ's with widths of $20-50 \mu \mathrm{m}$ as a function of the magnetic field. They interpreted that this is due to the coherent flow of the triangular JV lattice. Subsequently, Koshelev ${ }^{13}$ and Machida ${ }^{14}$ explained the oscillation by calculating the JV flow resistance by taking the surface barrier effect into consideration. In this model, since the surface barrier introduced by the boundary condition at the junction edges only influences the positions of JV's close to the surfaces, a bulk property (triangular JV lattice) is probed by the surface current. Reducing the junction width to $\sim \mu \mathrm{m}$ is expected to make the surface barrier effect compete with the bulk inductive coupling between the JV arrays in neighboring layers and result in drastically different phenomena. In smaller junctions $<10 \mu \mathrm{m}$, the authors found oscillations with the doubled period $\phi_{0}$ at high magnetic fields, ${ }^{15}$ and Hatano et $a l$. reported the $\phi_{0}$ oscillation in a submicron junction. ${ }^{16} \mathrm{Mo}-$ tivated by these results, Machida ${ }^{17}$ and Koshelev ${ }^{18}$ proposed advanced calculations that the triangular and the square JV lattices appear alternatively as a function of magnetic field and that the square lattice is more favorable for higher magnetic field and in samples with smaller widths. Since the square JV lattice is a result of in-phase locked oscillation of the Josephson currents of the stacked IJJ's, realization of a square JV structure has been considered as a central issue for an electromagnetic radiation from IJJ. Although the emission of terahertz electromagnetic waves from IJJ's in the absence of applied magnetic field ${ }^{19,20}$ is attributed to the sizerestricted cavity resonance of the rather large mesas $(\sim 100 \mu \mathrm{m})$, tuning excited wavelength by JV lattice like a field tunable photonic crystal $^{11,21}$ is a next goal of the IJJ emitter.

In this Brief Report, we present the experimental results of the JV flow resistance and $I V$ characteristics in Bi2212 microstructured single crystals with dimensions of 1-6 $\mu \mathrm{m}$ more clearly and systematically than in the previous work..$^{15}$ In the smaller samples and at higher fields, we found periodic oscillation of the JV flow resistance with the period of the $\phi_{0}$ instead of $\phi_{0} / 2$ as argued by the theories. The results clearly indicate that the crossover of the two oscillations is well tuned by both the superconducting anisotropy parameter and the junction size, which may prove formation of the square JV lattice in the static and slowly moving dynamic states.

Bi2212 single crystals grown with the traveling solvent floating zone method were fabricated into bridge-shaped mesoscopic junctions with a focused ion beam (FIB) machine SMI2050MS (SII NanoTechnology Inc.). ${ }^{22}$ The fabrication details are described in Ref. 23. Here, we report the results of seven junctions obtained from different slightly overdoped crystals as listed in Table I. $T_{c}$ 's are in the range of $84-90 \mathrm{~K}$. External magnetic fields were applied by split pair superconducting magnets that generate a horizontal magnetic field up to $80 \mathrm{kOe}$. Rotating the magnetic field around the $a b$ plane with a high precision rotator, $c$-axis resistance suddenly increases in the vicinity of the $a b$ plane..$^{15}$ This resistance is attributed to the JV flow resistance, and the lock-in state 
TABLE I. List of samples. $L, W$, and $t$ denote length $(\perp \boldsymbol{H})$, width $(\| \boldsymbol{H})$, and thickness $(\| c)$ of junctions, respectively. Oscillation starting field $H_{s}$ and crossover field $H_{1 / 2}$ were directly given by measurements. $\gamma$ was derived from $H_{1 / 2}$.

\begin{tabular}{lccccccc}
\hline \hline & $L(\mu \mathrm{m})$ & $W(\mu \mathrm{m})$ & $t(\mu \mathrm{m})$ & $H_{p}(\mathrm{kOe})$ & $H_{s}(\mathrm{kOe})$ & $H_{1 / 2}(\mathrm{kOe})$ & $\gamma$ \\
\hline B19 & 1.9 & 11.7 & 0.5 & 6.7 & 15 & 16 & 119 \\
E44 & 4.4 & 9.5 & 0.15 & 3.04 & 6.0 & 34 & 130 \\
H41 & 4.1 & 5.5 & 2.0 & 3.24 & 7.0 & 42 & 114 \\
H55 & 5.5 & 4.1 & 2.0 & 2.49 & 5.3 & 35 & 142 \\
P52 & 5.2 & 3.5 & 0.36 & 2.43 & 5.0 & 30 & 155 \\
Q50 & 5.0 & 4.9 & 0.16 & 2.60 & 5.3 & 46 & 121 \\
V31 & 3.1 & 5.1 & 1.0 & 4.11 & 7.6 & 33 & 173 \\
\hline \hline
\end{tabular}

without pancake vortices is realized in this angle range. We set the field alignment parallel to the $a b$ plane with an accuracy of 0.01 degree.

Figures 1(a) and 1(b) represent the magnetic-field dependencies of zero-current differential $c$-axis resistivity $\rho_{c}$ $=L W / t \cdot d V /\left.d I\right|_{I=0}$ at $60 \mathrm{~K}$ in sample B19 and P52, respectively. The data were derived from $I V$ characteristics measured under external magnetic fields parallel to the $a b$ plane changed step by step. This method for evaluation of the JV feature is less ambiguous than the method with the Ohmic resistance $V / I$. The $\rho_{c}$ in P52 smoothly increases below 7

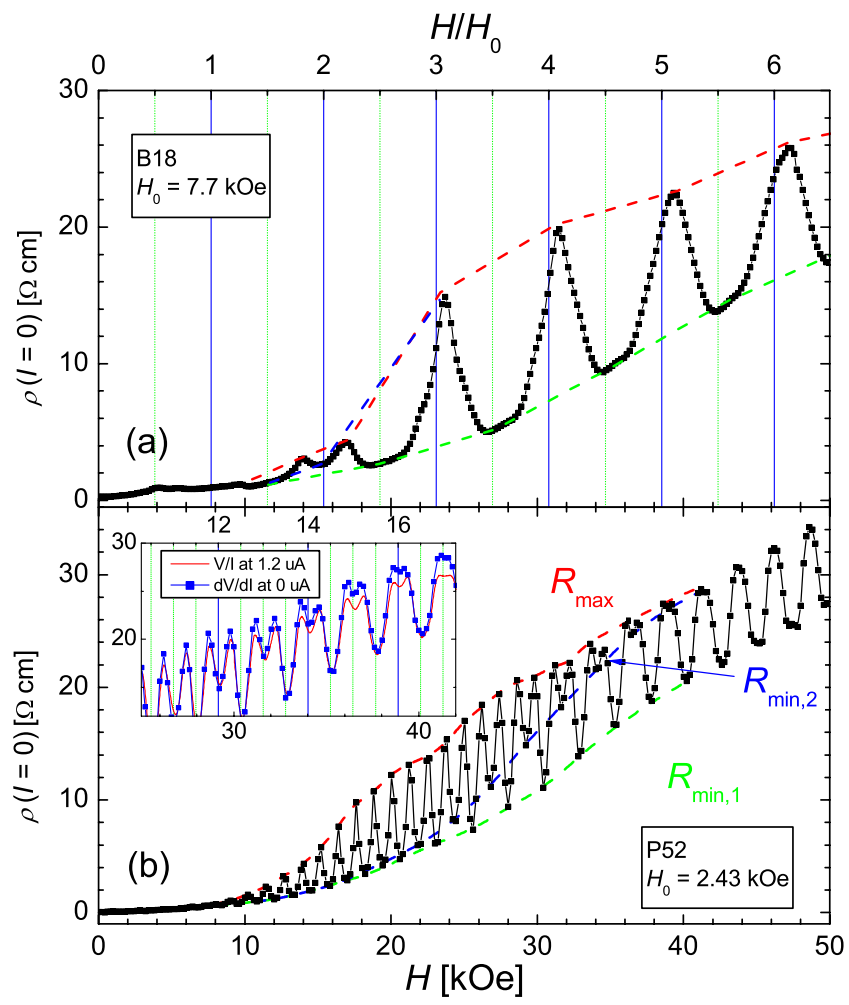

FIG. 1. (Color online) Field dependence of differential $c$-axis resistivity in B19 (a) and P52 (b) at $60 \mathrm{~K}$. Solid symbols represent differential resistance $d V / d I$ obtained from $I V$ characteristics. Broken curves connect maxima and minima points in $\rho(H)$. In the inset of (b), solid curve indicates Ohmic resistances $V / I$ obtained by sweeping magnetic field at $I=1.2 \mu \mathrm{A}$. Blue vertical grids in (a) and inset of (b) indicate that the fields of $H / H_{0}$ are integers.
kOe with increasing field from zero and then begins to oscillate with an oscillation center monotonically increasing, as shown in Fig. 1(b). Oscillation period $H_{p}$ is $1.2 \mathrm{kOe}$, which approximately coincides with adding a half flux quantum $\phi_{0} / 2$ to a JV array in the block layer as $H_{0} / 2=\phi_{0} / 2 L s$ $=1.3 \mathrm{kOe}$ for P52, where $s=15 \AA$ is the periodicity of the $\mathrm{CuO}_{2}$ double layers of $\mathrm{Bi} 2212$. This oscillation is quite similar to the previous results. ${ }^{12}$ The boundary effect for the triangular JV lattice is considered to be enhanced when the number of JV's in a layer corresponds to either integer $\left(H / H_{0}=k\right.$; full-integer matching) or half odd integer $\left(H / H_{0}\right.$ $=k+1 / 2$; half-integer matching) due to matching of the periodicity of the JV lattice with the junction width for both two cases. These two matchings yield the oscillation with period of $H_{0} / 2$ and the bottom at $H / H_{0}=k$ and $k+1 / 2$.

With increasing magnetic field, the full-integer matching was diminished above $25 \mathrm{kOe}$ and finally disappears above $40 \mathrm{kOe}$, while the half-integer matching does not change to $50 \mathrm{kOe}$. As a result, $H_{p}$ becomes $2.43 \mathrm{kOe}$, which is twice the value of the $H_{p}$ below $35 \mathrm{kOe}$ and close to the $H_{0}$ value of $2.50 \mathrm{kOe}$. This crossover from $\phi_{0} / 2$ to $\phi_{0}$ oscillation was observed in a lower field region in a sample with smaller $L$. Figure 1(a) shows a $\rho_{c}-H$ curve in B19 with $L=1.9 \mu \mathrm{m} . \phi_{0}$ oscillation was certainly observed above $20 \mathrm{kOe}$, and only a small dip at $H / H_{0}=2$ was found as a remnant of the $\phi_{0} / 2$ oscillation, as shown in the inset of Fig. 1(a).

Since linear $I V$ characteristics were observed even in the low current limit as displayed in Fig. 2(a), it was implied that there is no true critical current in IJJ with JV lattices. This makes a remarkable contrast with an ordinary vortex system under the $c$-axis magnetic field for HTSC, where a superlinear increase in voltage in $I V$ characteristics is regarded as the critical current. In order to compare with theoretically derived field dependencies of critical current density $J_{c}(H)$, the current $I_{0}(H)$ at which the induced voltages along the junction are $2 \mathrm{mV}$ is plotted as a function of magnetic field at various temperatures in Fig. 2(b). At $75 \mathrm{~K}$ below $15 \mathrm{kOe}$, $I_{0}(H)$ oscillates with a period of $H_{0} / 2$ as proposed in Ref. 13 for $J_{c}(H)$, while $I_{0}(H)$ above $35 \mathrm{kOe}$ shows oscillation with the period being $H_{0}$ as well as $\rho_{c}(H)$. The behavior of $I_{0}(H)$ in the high field region turns to be similar to $J_{c}(H)$ in a single Josephson junction (Fraunhofer pattern) that has local maxima at fields corresponding to $(k+1 / 2) \phi_{0}$. Such a Fraunhofer oscillation in $J_{c}(H)$ of multilayered system like Bi2212 is expected when condition $L \ll \lambda_{J} \equiv \gamma s$ is satisfied. ${ }^{7}$ How- 

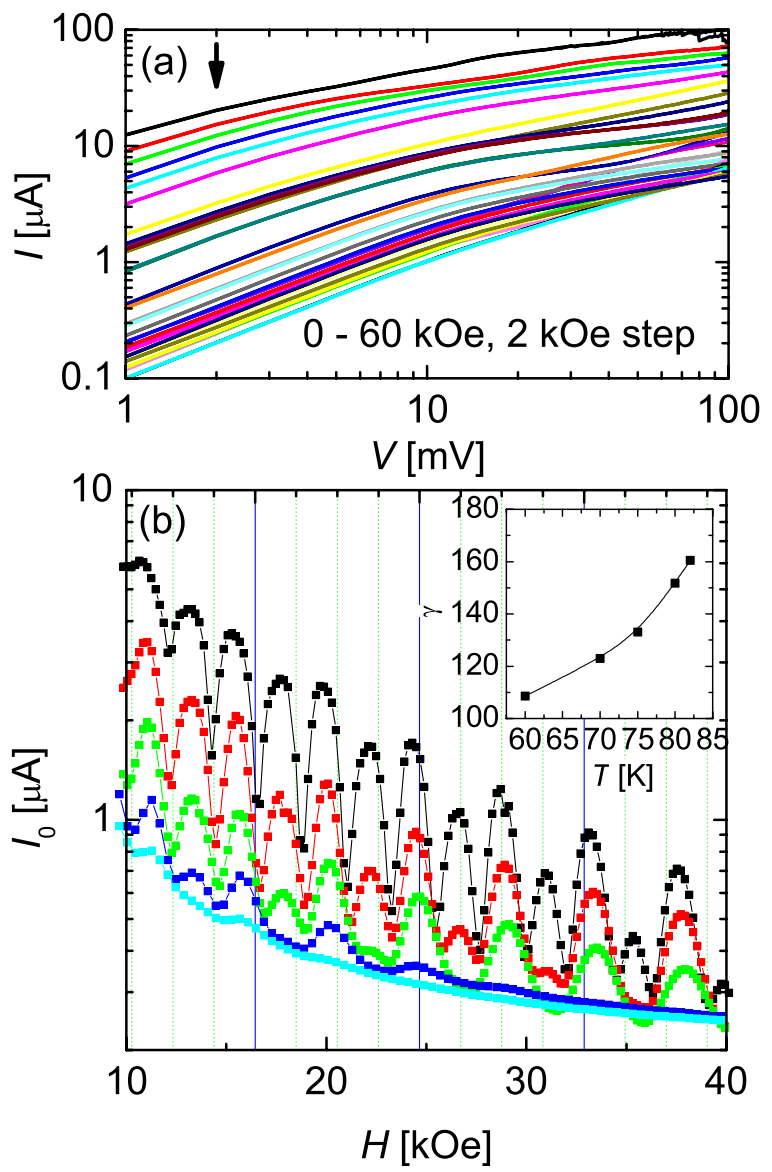

FIG. 2. (Color online) (a) $I V$ characteristics at $60 \mathrm{~K}$ in V31 $\left(T_{c}=84 \mathrm{~K}\right)$ at various magnetic fields from 0 (top) to $60 \mathrm{kOe}$ (bottom). (b) Field dependence of $I_{1}$, current at $V=2 \mathrm{mV}$ [the arrow in (a)], at $60,75,80$, and $82 \mathrm{~K}$ from top to bottom. Inset shows temperature dependence of anisotropy parameter $\gamma$ obtained by Eq. (1).

ever, $\lambda_{J}$ is estimated to be approximately $0.2 \mu \mathrm{m}$ for $\gamma$ $=100-200$, which is much smaller than the $L$ of these samples.

The experimental results demonstrate that the $\phi_{0}$ oscillation is a phenomenon induced by the external magnetic fields, thus it is important to consider competition between the bulk and the boundary effects for JV's. At relatively low fields where JV's start to form dense triangular lattices, the JV positions are mostly determined by the sharing interaction between vortices in adjacent layers due to the inductive coupling between layers. With increasing magnetic field, vortices inside the layer become closer and interaction between intralayer JV's is pronounced. The JV system is finally dominated by the surface deformation energy that lets JV's align in phase and turns toward the square lattice in the high field region at the cost of sharing energy between JV arrays. Since the sharing energy of JV's in a smaller junction with less JV's is smaller while the surface deformation does not depend on $L$, the $\phi_{0}$ oscillation is observed at a lower field. The single-junction-like behaviors are more pronounced in samples with shorter $L$ and at higher $H .^{13}$

The crossover between the triangular and the square JV lattices is quantitatively treated by Koshelev. ${ }^{18} \mathrm{He}$ derived

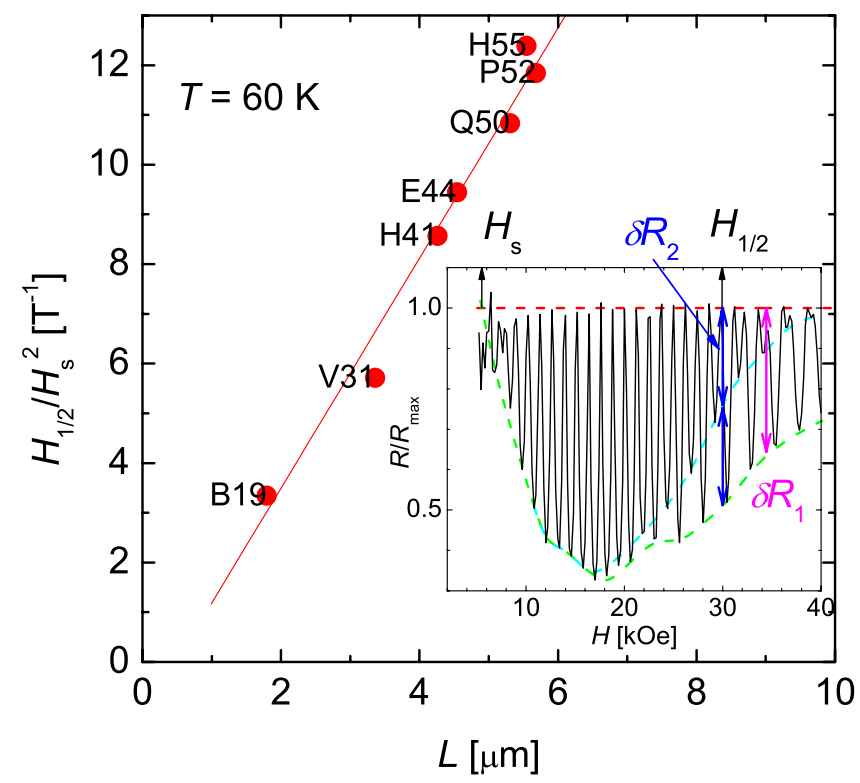

FIG. 3. (Color online) $L$ dependence of crossover field $H_{1 / 2}$ in which $\gamma$ is renormalized. Inset: method to extract $H_{s}$ and $H_{1 / 2}$. Solid and broken curves are those in Fig. 1(b) normalized by $R_{\max }$ [red (dark gray) broken curve].

the amplitudes of the resistance oscillation due to the halfinteger matching and the full-integer matching, $\delta R_{1}$ and $\delta R_{2}$, as functions of magnetic field. The magnetic field at $\delta R_{2} / \delta R_{1}=1 / 2$ is given by the junction length $L$ and the anisotropy parameter $\gamma$ as

$$
H_{1 / 2}=1.302 \frac{\phi_{0} L}{2 \pi \gamma^{2} s^{3}} .
$$

In order to extract generic boundary effect from our results in many samples with various oxygen concentrations, the sample specific parameter $\gamma$ should be taken from measurable parameters. The comparison between the $a b$-plane resistance before FIB fabrication the $c$-axis resistance ${ }^{24}$ does not work in this case because oxygen would be reduced during FIB fabrication in the vacuum and implanted gallium ions may damage the junction. These influences are more serious in junctions with widths less than $5 \mu \mathrm{m}$. To obtain the anisotropy parameter in the exactly same situation as the sample, we use the formation of the dense triangular JV lattice, which is considered necessary for $\phi_{0} / 2$ oscillation. Using a minimum field for triangular dense JV lattice $H_{\text {tr }}$ $=1.33 \phi_{0} / 2 \pi \gamma s^{2}, 25$ we obtain a simple relation:

$$
H_{1 / 2} / H_{\mathrm{tr}}^{2}=0.736 \frac{2 \pi s L}{\phi_{0}}=3.35[1 / \mu \mathrm{m} \mathrm{T}] L[\mu \mathrm{m}],
$$

where the magnetic fields are given in the unit of tesla. Assuming that $H_{\text {tr }}$ corresponds to $H_{s}$, the bare boundary effect for JV's may be extracted from the results.

Figure 3 shows the values of $H_{1 / 2} / H_{s}^{2}$ as a function of junction length $L$. $H_{1 / 2} / H_{s}^{2}$ is roughly proportional to $L$. $H_{1 / 2}$ and $H_{s}$ were obtained by extracting $\delta R_{1}$ and $\delta R_{2}$ from the resistance minima and maxima, as shown in the inset of Fig. 3. $\delta R_{1}$ and $\delta R_{2}$ are given by subtracting $R_{\min , 1}$, the smooth 
curves connecting resistance minima at $H / H_{0}=k+1 / 2$, and $R_{\text {min,2 }}$ from $R_{\max }$ in $\rho_{c}(H)$, respectively. Since it is difficult to decide the onset of the oscillation, $H_{s}$ was defined as $\delta R_{1}$ $=0$ by extrapolating $R_{\min , 1} / R_{\max }$ to 1 , so that $H_{s}$ may include more ambiguity than $H_{1 / 2}$. The slope of the fitted line is slightly smaller than Eq. (2) with a factor of 0.68 , meaning $H_{s}=1.2 H_{\text {tr }}$. Below $H_{\text {tr }} \mathrm{JV}$ lattices incommensurate along the $c$ axis are expected in the bulk as results of the sharing transitions from the triangular JV lattice. ${ }^{25}$ Considering the boundary effect which may suppress the sharing transition, the deviation could be reasonable. The result that $H_{1 / 2}$ can be scaled by $\gamma$ suggests that the crossover between $\phi_{0} / 2$ and $\phi_{0}$ oscillations is a result of competition between bulk sharing energy and the edge boundary effect for JV's. The argument that the $\phi_{0}$ oscillation is attributed to the Fiske step at higher bias current ${ }^{26}$ is not supported because $d V / d I$ at $I=0$ shows almost same behavior as Ohmic resistance $V / I$, as shown in the inset of Fig. 1(b). Therefore such a nonlinear effect in voltage should not be considered in this current region.

The temperature dependence of the crossover field is also consistent with the preceding discussions. As temperature is increased, $H_{1 / 2}$ considerably shifts to a lower field from 60 to $82 \mathrm{~K}$ as shown in Fig. 2. We derived $\gamma$ with Eq. (1) and plotted it in the inset of Fig. 2(b) as a function of temperature. It has been reported that $\gamma$ drastically increases in the vicinity of $T_{c}{ }^{27,28}$ It was also found that the amplitude of the oscillation of both $\rho_{c}(H)$ and $I_{0}(H)$ is maximized at $60-70 \mathrm{~K}$. This can be understood that the synchronization of stacked junctions is optimized at the temperatures: thermal fluctuation below $60 \mathrm{~K}$ smears the inhomogeneities contained in the crystal and helps form regular JV lattice, but thermal fluctuation above $70 \mathrm{~K}$ smears the matching conditions.

In summary, we investigated the Josephson vortex lattice structure in mesoscopic $(\sim \mu \mathrm{m})$ IJJ's with a probe of the JV flow resistance along the $c$ axis. The $c$-axis resistivity $\rho_{c}$ oscillates as a function of applied magnetic field with a oscillation period changing from $\phi_{0} / 2$ to $\phi_{0}$, which indicates triangular JV lattice and a possible square JV lattice, respectively. Crossover field $H_{1 / 2}$ linearly decreases with junction length $L$. This is attributed to the interplay between the boundary effect and the inductive coupling between JV arrays in IJJ's.

We thank M. Machida and A. Koshelev for their fruitful discussions and critical comments. This work was partially supported by a Grant-in-Aid for Young Scientists (B) and the Core-to-Core Program "Nanoscience and Engineering in Superconductivity" by the Japan Society for the Promotion of Science (JSPS).

\footnotetext{
*kakeya@kuee.kyoto-u.ac.jp

${ }^{1}$ R. Kleiner, F. Steinmeyer, G. Kunkel, and P. Müller, Phys. Rev. Lett. 68, 2394 (1992).

${ }^{2}$ G. Oya, N. Aoyama, A. Irie, S. Kishida, and H. Tokutaka, Jpn. J. Appl. Phys., Part 2 31, L829 (1992).

${ }^{3}$ S. Sakai, P. Bodin, and N. F. Pedersen, J. Appl. Phys. 73, 2411 (1993).

${ }^{4}$ T. Koyama and M. Tachiki, Phys. Rev. B 54, 16183 (1996).

${ }^{5}$ I. Kakeya, K. Kindo, K. Kadowaki, S. Takahashi, and T. Mochiku, Phys. Rev. B 57, 3108 (1998).

${ }^{6}$ A. A. Yurgens, Supercond. Sci. Technol. 13, R85 (2000).

${ }^{7}$ L. N. Bulaevskii, J. R. Clem, and L. I. Glazman, Phys. Rev. B 46, 350 (1992).

${ }^{8}$ X. Hu and M. Tachiki, Phys. Rev. Lett. 85, 2577 (2000).

${ }^{9}$ G. Hechtfischer, R. Kleiner, K. Schlenga, W. Walkenhorst, P. Müller, and H. L. Johnson, Phys. Rev. B 55, 14638 (1997).

${ }^{10}$ V. M. Krasnov, N. Mros, A. Yurgens, and D. Winkler, Phys. Rev. B 59, 8463 (1999).

${ }^{11}$ I. Kakeya, T. Wada, R. Nakamura, and K. Kadowaki, Phys. Rev. B 72, 014540 (2005).

${ }^{12}$ S. Ooi, T. Mochiku, and K. Hirata, Phys. Rev. Lett. 89, 247002 (2002).

${ }^{13}$ A. E. Koshelev, Phys. Rev. B 66, 224514 (2002).

${ }^{14}$ M. Machida, Phys. Rev. Lett. 90, 037001 (2003).

${ }^{15}$ I. Kakeya, M. Iwase, T. Yamamoto, and K. Kadowaki, arXiv:cond-mat/0503498 (unpublished).
}

${ }^{16}$ S. Urayama, T. Hatano, H. B. Wang, M. Nagao, S. M. Kim, and J. Arai, arXiv:cond-mat/0602659 (unpublished).

${ }^{17}$ M. Machida, Phys. Rev. Lett. 96, 097002 (2006).

${ }^{18}$ A. E. Koshelev, Phys. Rev. B 75, 214513 (2007).

${ }^{19}$ L. Ozyuzer, A. Koshelev, C. Kurter, N. Gopalsami, Q. Li, M. Tachiki, K. Kadowaki, T. Yamamoto, H. Minami, H. Yamaguchi et al., Science 318, 1291 (2007).

${ }^{20}$ K. Kadowaki, H. Yamaguchi, K. Kawamata, T. Yamamoto, H. Minami, I. Kakeya, U. Welp, L. Ozyuzer, A. Koshelev, C. Kurter et al., Physica C 468, 634 (2008).

${ }^{21}$ S. Savel'ev, A. L. Rakhmanov, and F. Nori, Phys. Rev. B 74, 184512 (2006).

${ }^{22}$ S.-J. Kim, Y. I. Latyshev, and T. Yamashita, Supercond. Sci. Technol. 12, 729 (1999).

${ }^{23}$ I. Kakeya, K. Fukui, K. Kawamata, T. Yamamoto, and K. Kadowaki, Physica C 468, 669 (2008).

${ }^{24}$ S. Yu, S. Ooi, T. Mochiku, and K. Hirata, Phys. Rev. B 76, 092505 (2007).

${ }^{25}$ Y. Nonomura and X. Hu, Phys. Rev. B 74, 024504 (2006).

${ }^{26}$ A. V. Ustinov and N. F. Pedersen, Phys. Rev. B 72, 052502 (2005).

${ }^{27}$ J. Mirkovic, S. E. Savel'ev, E. Sugahara, and K. Kadowaki, Phys. Rev. B 66, 132505 (2002).

${ }^{28}$ M. Konczykowski, C. J. van der Beek, A. E. Koshelev, V. Mosser, M. Dodgson, and P. H. Kes, Phys. Rev. Lett. 97, 237005 (2006). 